\title{
Parameter estimation procedure based on input-output integro-differential polynomials. Application to the Hindmarsh-Rose model.
}

\author{
Nathalie Verdière $^{1}$ and Carine Jauberthie ${ }^{2}$
}

\begin{abstract}
This paper deals with a parameter estimation method based on input-output integro-differential polynomials. From the Rosenfeld-Groebner algorithm, some differential relations depending only on the inputs, the outputs and the parameters of the model are obtained. A pretreatment consisting in some integrations of these relations permits to obtain new ones. The latter contain essentially integrals depending only on the model inputs/outputs and their higher order derivatives are lower than the initial relations. Therefore, they are less sensitive to the noise on the measurements compared to the initial ones. Integrating permits also to attenuate the effect of the noise improving by the same the parameter estimates. However, even if the numerical estimation algorithm provides a very good value of the parameters, the latter can lead to an incorrect behavior of the model output. Indeed, in biological or physical applications, a little change of some parameter values can lead to a radical change of the model behavior as for the Hopf bifurcation. A Hopf bifurcation refers to a radical change of the model output dynamic due to a parameter crossing a reference value. Therefore, an algorithm is proposed in this paper to test the reliability of any parameter estimation procedure with respect to the dynamic of the system. More precisely, from a given noise on the output(s), it consists in calculating the probability that the result of a parameter estimation algorithm will permit to reproduce the correct behavior of the model output. Finally, this algorithm is applied on the estimation procedure based on the input-output integrodifferential polynomials and on the Hindmarsh-Rose model, a slow-fast model able to reproduce the main behaviors of a neuron and presenting a Hopf bifurcation.
\end{abstract}

\section{INTRODUCTION}

Parameter estimation of dynamical systems is an important step in the modeling to make a model usable. Several global or local methods can be found in the literature [11], [18]. They are mainly focused on the optimization of some criterion function over the parameter space and required a first initial guess or at least a definition interval. This first initial guess can be obtained by an industrial process or by a numerical procedure and may determine the convergence of a local parameter estimation algorithm. This paper proposes to focus on a parameter estimation method based on differential polynomials in order to obtain a first initial guess of the parameters without, a priori, any knowledge of them.

\footnotetext{
*This work was not supported by any organization

${ }^{1}$ Verdière $\mathrm{N}$. is with University of Normandie, UNIHAVRE, LMAH, FR-CNRS-3335, ISCN, 76600 Le Havre, France verdiern@univ-lehavre.fr

${ }^{2}$ Jauberthie C. is with LAAS-CNRS, Université de Toulouse, CNRS, UPS, 7 avenue du Colonel Roche, 31400 Toulouse, France cjaubertelaas.fr
}

Afterwards, the general dynamical systems have the following form:

$$
\Gamma^{\theta}\left\{\begin{array}{l}
\dot{x}(t, \theta)=f(x(t, \theta), \theta)+u(t) g(x(t, \theta), \theta), \\
y(t, \theta)=h(x(t, \theta), \theta) .
\end{array}\right.
$$

Here $x(t, \theta) \in \mathbb{R}^{n}$ denotes the state variables. The model outputs are given by $y(t, \theta) \in \mathbb{R}^{m}$. The set of model parameters to be estimated are given by $\theta \in \mathcal{U}_{p}$ the unknown parameter vector $\left(\mathcal{U}_{p}\right.$ is an open subset in $\left.\mathbb{R}^{s}\right)$. The functions $f(., \theta), g(., \theta)$ and $h(., \theta)$ are real, rational and analytic for every $\theta \in \mathcal{U}_{p}$ on $M$ (a connected open subset of $\mathbb{R}^{n}$ such that $x(t, \theta) \in M$ for every $\theta \in \mathcal{U}_{p}$ and every $t \in[0, T]) . u(t) \in \mathbb{R}^{l}$ denotes the input variables. In the case of uncontrolled system, $u$ is equal to 0 . We also let $x_{0}=\left(x_{0, i}\right)_{i=1, \ldots, n}$ the vector of initial conditions for $x(t, \theta)$ whose some components can depend on the parameters to be estimated.

From system $\Gamma^{\theta}$, some differential relations can be obtained in using the Rosenfeld-Groebner elimination algorithm and are called input-output (IO) polynomials [2]. From the latter, an identifiability study of $\Gamma^{\theta}$ can be done and is recommended before putting in place a parameter estimation procedure. Indeed, this study ensures that the parameters can be uniquely inferred from the output measurements of the system. However, IO polynomials may contain sometimes high-order derivatives which is a factor of instability in numerical procedures. Indeed, estimate derivatives from noisy measurements is an ill-posed problem. The main challenge is also to obtain new relations with lower order derivatives. Lots of authors have proposed different ways to obtain integro-differential input-output (ID-IO) relations since they are less sensitive to the noise compared to the initial IO polynomials (for example [1], [12], [14], [17]). Indeed, ID-IO polynomials may contain lower order derivatives and integrating permits to attenuate the structured noise whose amplitude is unknown [6]. In order to obtain ID-IO polynomials, [12] multiplied them by modulating functions and then integrate the new relation whereas [14] used iterative integrals in the frequency domain. In [17], a distribution approach was proposed with a complete study on the numerical error due to this method. In this paper, for the parameter estimation, we focus on the simple use of iterative integrals leading to relations with no derivative in our case.

Even in the case of a good parameter estimate, measurement noise is an important factor of uncertainty and error in 
parameter estimation procedures. To have the probability that the parameter estimate captures the dynamic of the system with respect to the noise on the output(s), an algorithm is proposed in this paper. To apply these results, the Hindmarsh-Rose (HR) model resulting from a simplification and a generalization of the Hodgkin-Huxley model is considered (see [7], [8] for more details). The aim of such models is to better understand neuron activity as presented in [9], [10], [16]. Its particularity is to present a Hopf bifurcation with respect to its slow-fast parameter. To estimate parameters of the HR neuron model, methods were already proposed. In [16], the authors give two approaches. The first one concerns the synchronization based parameter estimation and consists in solving a least-square problem subjects to constraints. The second approach is based on the adaptive observers as in [15]. This method aims to find a dynamical system so that it synchronizes with the measured voltages from a real neuron. In [13], authors use nonlinear optimization exploiting the particular structure of the relevant cost function. However, for main of these methods, a first initial guess is needed. That is why, we propose to use the approach based on ID-IO polynomials to obtain this first initial guess.

The paper is organized as follows. In Section II, the parameter estimation procedure based on the ID-IO polynomials is presented as well as the procedure to calculate the probability that a parameter estimate will permit to reproduce the correct behavior of the model output with respect to the noise. Section III concerns the HR model. Its first part presents this slow-fast model and its bifurcation. In the second part, an identifiability study and a parameter estimation are achieved on it. The end of Section III ends with an analysis of the effect of the noise on the estimation of its bifurcation parameter. Finally, Section IV concludes the paper.

\section{PARAMETER ESTIMATION METHOD}

\section{A. Differential Algebra and identifiability}

An identifiability study ensures that two different parameter vectors will lead to two different model outputs. The definition can be formalized as follows:

Definition 1: The model $\Gamma^{\theta}$ is said globally identifiable at $\theta$ if a time $t_{1} \in[0, T]$ exists such that for all $t \in\left[0, t_{1}\right]$, for all $\tilde{\theta} \in \mathcal{U}, \tilde{\theta} \neq \theta$ then $y(t, \theta)$ and $y(t, \tilde{\theta})$ are different.

The identifiability study can be analyzed through relations linking input, output and parameters of model $\Gamma^{\theta}$. They are obtained using the Rosenfeld-Groebner algorithm implemented in the package DifferentialAlgebra of Maple [2]. From the elimination order consisting in eliminating unobservable variables, the algorithm provides IO polynomials having the following form (See [5]):

$$
P_{i}(y, u, \theta)=m_{0}^{i}(y, u)+\sum_{j=1}^{q_{i}} \gamma_{j}^{i}(\theta) m_{j, i}(y, u)=0
$$

where $\left(\gamma_{j}^{i}\right)_{1 \leq j \leq q_{i}}$ are rational in $\theta, \gamma_{u}^{i} \neq \gamma_{v}^{i}(u \neq v)$, $\left(m_{j, i}\right)_{1 \leq j \leq q_{i}}$ are differential polynomials with respect to $y$ and $u . m_{0}^{i} \neq 0$ and $i$ from 1 to $m$.

According to [5], the number of relations is the number of observations.

Afterwards, to lighten the notations, only one output is considered so that the index $i$ is omitted. Let us denote $s$ the time derivative of highest order of $y$ in $P$.

From the coefficients of the IO polynomial $P$ and the initial conditions (if any), we can define the real-valued function $\phi$ by $\phi: \theta=\left(\theta_{1}, \ldots, \theta_{p}\right) \mapsto$ $\left(\gamma_{1}, \ldots, \gamma_{q}, y(0, \theta), \ldots \ldots, y^{(s-1)}(0, \theta)\right)$. Under some technical conditions omitted in this paper (for more details, the interested reader can refer to [5]), the identifiability result is the following:

Proposition 1: Assume that the functional determinant $\triangle P(y, u, \theta)=\operatorname{det}\left(m_{j}(y, u), j=1, \ldots, q\right) \not \equiv 0^{1}$ and the coefficient of $y^{(s)}$ is not equal to zero at $t=0$. The model $\Gamma^{\theta}$ is globally identifiable if and only if the function $\phi$ is injective on $\mathcal{U}_{p}$.

\section{B. Estimation procedure}

The measurement noise is taken into account by assuming that the output $y$ is disturbed by a centered Gaussian noise $\eta$ so that the measurement $z$ of $y$ has the following form: $z(t)=y(t, \bar{\theta})+\eta(t)$ where $\bar{\theta}$ represents the "true" parameter vector value. If $\left(t_{k}\right)_{1 \leq k \leq N}$ is a sequence of discrete times, we denote $\left\{z_{k}=z\left(t_{k}\right), k=1, \ldots, N\right\}$ the set of measurements at $\left(t_{k}\right)_{1 \leq k \leq N}$ and $u_{k}=u\left(t_{k}\right)$ the associated inputs.

Let $f$ a real-valued function and $I_{\nu}(f), \nu \in \mathbb{N}$, the integrated function such that

$$
I_{\nu}(f)=\int_{t-\tau}^{t} \int_{\tau_{1}-\tau}^{\tau_{1}} \ldots \int_{\tau_{\nu-1}-\tau}^{\tau_{\nu}} f\left(\tau_{\nu}\right) d \tau_{\nu} \ldots d \tau_{1} .
$$

Its approximated value by a numerical procedure from the measurements $z_{k}, k=1, \ldots, N$ will be denoted $I_{\nu}^{\text {est }}(f)$. Due to the linearity of the integral, a new relation is obtained from $P$ and can be rewritten:

$$
I_{\nu}(P)=I_{\nu}\left(\tilde{m}_{0}(y, u)\right)+\sum_{j=1}^{q} \tilde{\gamma}_{j}(\theta) I_{\nu}\left(\tilde{m}_{j}(y, u)\right) .
$$

Afterwards, $I_{\nu}(P)$ is called the ID-IO polynomial.

Evaluating the expression $I_{\nu}(P)$ at each $t_{k}$ leads to a linear system whose unknown are the coefficients $\tilde{\gamma}_{j}(\theta), j=$ $1, \ldots, q$. Indeed, $I_{\nu}(P)$ is linear with respect to the parameter blocks $\tilde{\gamma}_{1}(\theta), \ldots, \tilde{\gamma}_{q}(\theta)$. The following system can also be deduced:

$$
A \tilde{\Theta}=b
$$

${ }^{1}$ To verify this assumption, it is sufficient to verify the linear independence of the $m_{j}(y, u), j=1, \ldots, q$ by checking the functional determinant given by the Wronskian [4]

$$
\left|\left(\begin{array}{ccc}
m_{1}(y, u) & \ldots & m_{q}(y, u) \\
m_{1}(y, u)^{(1)} & \cdots & m_{q}(y, u)^{(1)} \\
& \ddots & \\
m_{1}(y, u)^{(q-1)} & \ldots & m_{q}(y, u)^{(q-1)}
\end{array}\right)\right|
$$

is not identically equal to zero. In fact, the monomials are linearly independent if there exists a time point at which the Wronskian is non-zero. 
where $\tilde{\Theta}=\left(\tilde{\gamma}_{1}(\theta), \ldots, \tilde{\gamma}_{q}(\theta)\right)^{T}$. The kth line of $A$ and $b$ are respectively given by $(A)_{k}=\left(I_{\nu}^{\text {est }}\left(\tilde{m}_{j}(y, u)\right)\right)_{j=1, \ldots, q}$ and $b_{k}=-I_{\nu}^{e s t}\left(\tilde{m}_{0}(y, u)\right)$.

Remark 1: The linear system that can be deduced directly from $P$ may contain derivatives of high order. Indeed, the elimination algorithm of Rosenfeld-Groebner is based on addition, differentiation in time and multiplication by any polynomials in $x, u, y$ and $\theta$. Or, integrating permits to decrease the order of some derivatives but also to attenuate the structured noise whose amplitude is unknown. By definition, a structured noise is solution of a given homogeneous linear differential equation (See [6] for more detailed). Finally, whatever the integral method applied on $P$, the new relation has always given better results in the case of the parameter estimation (See [12], [14], [17] for some examples). Remark that the estimated parameters obtained by this way may be used as an initial guess for a local algorithm to improve the results.

Even in the case of a good relative error between the exact and the estimated parameters, the behavior of the output and the simulated output may not be the same. For example, if the model has a Hopf bifurcation, near the critical point, the model output can either tend to a stable equilibrium point or be periodic. Thus, the estimated parameter vector can lead to a deterioration of the dynamic of the model output during the time as it will be seen at section III-C. Remark too that we can not guarantee the result obtained by a local algorithm with a first initial guess for which the system does not present the good dynamic (see Remark 2). In order to test the reliability of any parameter estimation procedure, we propose, in the following section, an algorithm to calculate the probability to have an estimated parameter providing the correct behavior of the model output.

\section{Probability of obtaining the correct behavior}

Let $\theta$ the parameter vector of Model $\Gamma^{\theta}$ to estimate. Suppose that its kth component, $\theta_{k}$, is a bifurcation parameter. With no restriction, we can suppose that $k=1$. Let $\theta_{1}^{c}$ its bifurcation value that is the value for which a little change leads to a complete different behavior of Model $\Gamma^{\theta}$. For a given error level, to determine the probability for the estimated value of $\theta_{1}$ not being in the accepted behavioral interval, 1000 simulated data are generated from the output model. For each simulated data, a parameter estimation procedure is used so that the parameter vector $\hat{\theta}$ containing the estimated value $\hat{\theta}_{1}$ of $\theta_{1}$ is calculated. The comparison between $\theta_{1}^{c}$ and $\hat{\theta}_{1}$ permits to conclude if $\hat{\theta}_{1}$ is in the accepted behavioral interval. The procedure is summed up below.

1) Use the nominal parameter values to numerically solve the ODE model to get the solution of the output or measurement variables at the experimental design time points.

2) Generate $M$ sets (e.g., 1000) of simulated data from the output or measurement model with a given measurement error level.

3) For each of the $M$ simulated data sets, estimate parameter vector $\hat{\theta}$ using the method developed at section II for example.

4) Count the number of estimates $\hat{\theta}$ that are in the accepted behavioral interval.

\section{HINDMARSH-ROSE MODEL}

The model of Hindmarsh-Rose (HR) results from a simplification and a generalization of the Hodgkin-Huxley model [7], [8]. This model is a slow-fast model and is able to reproduce the rich dynamics of a neuron, such as spiking, bursting and chaotic behaviors. The aim of such models is to better understand neuron activity [9], [10], [16]. The HR model [8] reads as follows (where $\theta=(a, b, d, \varepsilon)^{T}$ ):

$$
\left\{\begin{aligned}
\dot{x}_{1}(t, \theta)= & x_{2}(t, \theta)+a x_{1}(t, \theta)^{2}-x_{1}(t, \theta)^{3} \\
& -x_{3}(t, \theta)+I(t) \\
\dot{x}_{2}(t, \theta)= & 1-d x_{1}(t, \theta)^{2}-x_{2}(t, \theta) \\
\dot{x}_{3}(t, \theta)= & \varepsilon\left(b\left(x_{1}(t, \theta)-c_{x_{1}}\right)-x_{3}(t, \theta)\right) .
\end{aligned}\right.
$$

$x_{1}$ represents the membrane potential and is the output $y:=x_{1}$ of the HR model, $x_{2}$ the recovery variable associated with the fast current due to the passage of the $\mathrm{Na}^{+}$or $\mathrm{K}^{+}$ ions and $x_{3}$ the adaptation current associated with the slow current due to the passage of the $\mathrm{Ca}^{+}$ions. Parameters $a, b$, $d$ and $\epsilon$ are experimentally determined from measurements of membrane potentials, while $c_{x_{1}}$ is the $x_{1}$-coordinate of the leftmost equilibrium of the two-dimensional system given by the first two equations of (3) when $I(t)=0$ and $x_{3}(t, \theta)=0$. I corresponds to the applied current (in Ampere) and afterwards it is supposed constant. Finally, parameter $\varepsilon$ represents the ratio of time scales between fast and slow fluxes across the membrane of a neuron.

The authors in [3] show that the parameter $\varepsilon$ presents a Hopf bifurcation. A Hopf bifurcation refers to the local birth or death of a periodic solution near an equilibrium point due to a change of one parameter value. Therefore, the HR model can present different dynamics with respect to the value of $\varepsilon$. Let $\varepsilon_{c}$ its bifurcation value. In [3], authors give an explicit formula of the bifurcation parameter $\varepsilon_{c}$ in function of $a, b$ and $d$. For example, for $a=3, b=4, d=$ $5, I(t)=3.25 \mathrm{~A}$, System (3) admits one equilibrium point and the constant bifurcation parameter $\varepsilon_{c}$ is approximatively equal to 0.125912 .

Fig. 1 and 2 represent the $\left(x_{1}, x_{2}, x_{3}\right)$ view of the phase portrait for two different values of $\varepsilon$, before and after the Hopf bifurcation. For $\varepsilon$ greater than $\varepsilon_{c}$ the solution is periodic and disappears when $\varepsilon$ increases such that the system has a stable equilibrium point. The time series of system (3) for the two previous parameters of $\varepsilon$ are represented at Fig. 3 and 4 respectively.

\section{A. Identifiability study of the HR model}

To obtain the IO polynomial of the HR model, the package DifferentialAlgebra of Maple is used.

$I$ being an input of the system, the elimination order $[\theta] \prec$ $[y, I] \prec\left[x_{1}, x_{2}, x_{3}\right]$ is chosen. It consists in eliminating $x_{1}$, $x_{2}, x_{3}$ in order to obtain a polynomial depending only on $y$, $\theta$ and $I$. The Rosenfeld-Groebner algorithm provides a polynomial with a derivative of order 3 and with 24 expressions. 


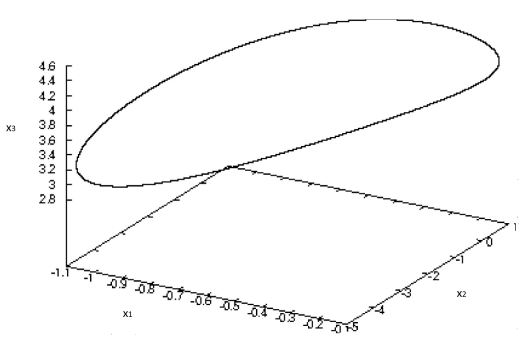

Fig. 1. $\left(x_{1}, x_{2}, x_{3}\right)$ view of the phase portrait of system (3) when $\varepsilon=$ $0.12<\varepsilon_{c}$.

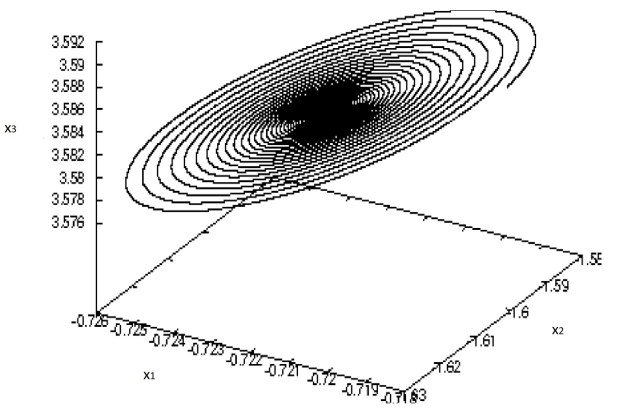

Fig. 2. $\left(x_{1}, x_{2}, x_{3}\right)$ view of the phase portrait of system (3) when $\varepsilon=$ $0.13>\varepsilon_{c}$.

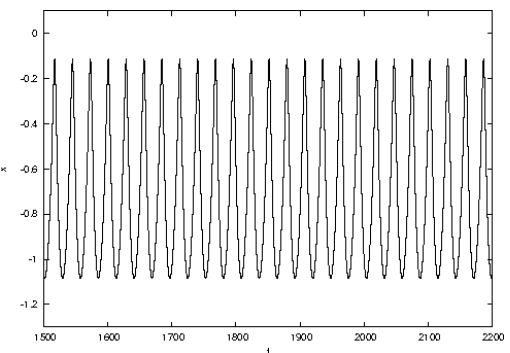

Fig. 3. Time series of system (3) when $\varepsilon=0.12<\varepsilon_{c}$.

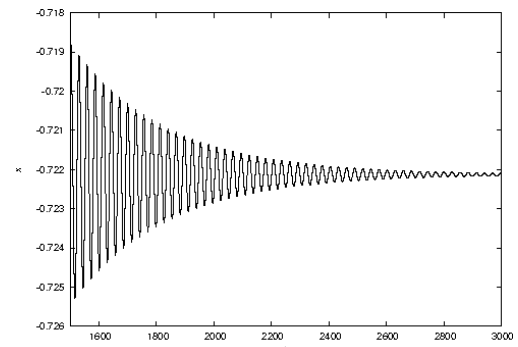

Fig. 4. Time series of system (3) when $\varepsilon=0.13>\varepsilon_{c}$.

To obtain a simpler polynomial, let $u_{1}(t)=e^{-t}, v_{1}(t)=$ $e^{-t} \int_{0}^{t} x_{1}^{2}(s, \theta) e^{s} d s$. In integrating the second Equation of (3), one gets $x_{2}(t, \theta)=\left(x_{2}(0, \theta)-1\right) u_{1}(t)+1-d v_{1}(t)$.
Consider now the following system:

$$
\left\{\begin{aligned}
\dot{x}_{1}(t, \theta)= & x_{2}(t, \theta)+a x_{1}^{2}(t, \theta)-x_{1}^{3}(t, \theta) \\
& -x_{3}(t, \theta)+I(t), \\
x_{2}(t, \theta)= & \left(x_{2}(0, \theta)-1\right) u_{1}(t)+1-d v_{1}(t), \\
\dot{x}_{3}(t, \theta)=\varepsilon\left(b\left(x_{1}(t, \theta)-c_{x_{1}}\right)-x_{3}(t, \theta)\right), & -u_{1}(t) \\
\dot{u}_{1}(t)=-v_{1}(t)+x_{1}^{2}(t) . &
\end{aligned}\right.
$$

System (3) completed with the initial conditions $\left(x_{1}(0, \theta), x_{2}(0, \theta), x_{3}(0, \theta)\right)$ is equivalent to System (4) completed with $\left(x_{1}(0, \theta), x_{2}(0, \theta), x_{3}(0, \theta), 1,0\right)$. Considering now the elimination order $[\theta]<\left[y, u_{1}, v_{1}, I\right] \prec\left[x_{1}, x_{2}, x_{3}\right]$, we obtain (the time variable $t$ is omitted):

$$
\begin{aligned}
P:= & \ddot{y}+3 y^{2} \dot{y}+\left(-\varepsilon y_{0}+\varepsilon+y_{0}-1\right) u_{1} \\
& +(d \varepsilon-d) v_{1}+\varepsilon\left(x^{3}+\dot{y}\right)+(-a \varepsilon+d) y^{2} \\
& -2 a y \dot{y}+b \varepsilon y-b c_{y} \varepsilon-\varepsilon(1+I)=0 .
\end{aligned}
$$

The function $\phi$ is defined by: $\phi: \theta \mapsto\left(-\varepsilon y_{0}+\varepsilon+y_{0}-\right.$ $\left.1, d \varepsilon-d, \varepsilon,-a \varepsilon+d,-2 a, b \varepsilon,-b c_{x} \varepsilon-\varepsilon(1+I)\right)$ is clearly injective so the model (3) with $y=x_{1}$ is identifiable.

Remark that $y_{0}$ can be estimated too using the polynomial $P$.

\section{B. ID-IO polynomial}

In order to decrease the derivative order of polynomial $P$ defined by (5), $P$ is integrated twice given the following relation (the variable $\theta$ is omitted):

$$
\begin{aligned}
& I_{2}(P):=\int_{t-\tau}^{t} \int_{\tau_{1}-\tau}^{\tau_{1}} \ddot{y}\left(\tau_{2}\right) d \tau_{2} d \tau_{1} \\
& +3 \int_{t-\tau}^{t} \int_{\tau_{1}-\tau}^{\tau_{1}} y\left(\tau_{2}\right)^{2} \dot{y}\left(\tau_{2}\right) d \tau_{2} d \tau_{1} \\
& +\left(-\varepsilon y_{0}+\varepsilon+y_{0}-1\right) \int_{t-\tau}^{t} \int_{\tau_{1}-\tau}^{\tau_{1}} u_{1}\left(\tau_{2}\right) d \tau_{2} d \tau_{1} \\
& +(d \varepsilon-d) \int_{t-\tau}^{t} \int_{\tau_{1}-\tau}^{\tau_{1}} v_{1}\left(\tau_{2}\right) d \tau_{2} d \tau_{1} \\
& -\varepsilon \int_{t-\tau}^{t} \int_{\tau_{1}-\tau}^{\tau_{1}}\left(-y\left(\tau_{2}\right)^{3}-\dot{y}\left(\tau_{2}\right)\right) d \tau_{2} d \tau_{1} \\
& +(-a \varepsilon+d) \int_{t-\tau}^{t} \int_{\tau_{1}-\tau}^{\tau_{1}} y\left(\tau_{2}\right)^{2} d \tau_{2} d \tau_{1} \\
& -2 a \int_{t-\tau}^{t} \int_{\tau_{1}-\tau}^{\tau_{1}} y\left(\tau_{2}\right) \dot{y}\left(\tau_{2}\right) d \tau_{2} d \tau_{1} \\
& +b \varepsilon \int_{t-\tau}^{t} \int_{\tau_{1}-\tau}^{\tau_{1}} y\left(\tau_{2}\right) d \tau_{2} d \tau_{1} \\
& -\left(I \varepsilon+b c_{y} \varepsilon+\varepsilon\right) \int_{t-\tau}^{t} \int_{\tau_{1}-\tau}^{\tau_{1}} 1 d \tau_{2} d \tau_{1} .
\end{aligned}
$$

Some double integrals can be simplified:

$$
\begin{gathered}
\int_{t-\tau}^{t} \int_{\tau_{1}-\tau}^{\tau_{1}} \dot{y}\left(\tau_{2}\right) d \tau_{2} d \tau_{1} \\
=\int_{t-\tau}^{t}\left(y\left(\tau_{1}\right)-y\left(\tau_{1}-\tau\right)\right) d \tau_{1}, \\
\int_{t-\tau}^{t} \int_{\tau_{1}-\tau}^{\tau_{1}} \ddot{y}\left(\tau_{2}\right) d \tau_{2} d \tau_{1}= \\
y(t)-2 y(t-\tau)+y(t-2 \tau),
\end{gathered}
$$




$$
\begin{gathered}
3 \int_{t-\tau}^{t} \int_{\tau_{1}-\tau}^{\tau_{1}} y\left(\tau_{2}\right)^{2} \dot{y}\left(\tau_{2}\right) d \tau_{2} d \tau_{1}= \\
\int_{t-\tau}^{t}\left(y\left(\tau_{1}\right)^{3}-y\left(\tau_{1}-\tau\right)^{3}\right) d \tau_{1}, \\
2 \int_{t-\tau}^{t} \int_{\tau_{1}-\tau}^{\tau_{1}} \dot{y}\left(\tau_{2}\right) y\left(\tau_{2}\right) d \tau_{2} d \tau_{1} \\
=\int_{t-\tau}^{t}\left(y\left(\tau_{1}\right)^{2}-y\left(\tau_{1}-\tau\right)^{2}\right) d \tau_{1}, \\
\int_{t-\tau}^{t} \int_{\tau_{1}-\tau}^{\tau_{1}} d \tau_{2} d \tau_{1}=\tau^{2} .
\end{gathered}
$$

In substituting these relations in the ID-IO polynomial (6), the following relation which does not contain no derivative is obtained:

$$
\begin{aligned}
& I_{2}(P)=y(t)-2 y(t-\tau)+y(t-2 \tau) \\
& +\int_{t-\tau}^{t}\left(y\left(\tau_{1}\right)^{3}-y\left(\tau_{1}-\tau\right)^{3}\right) d \tau_{1} \\
& +\left(-\varepsilon y_{0}+\varepsilon+y_{0}-1\right) \int_{t-\tau}^{t} \int_{\tau_{1}-\tau}^{\tau_{1}} u_{1}\left(\tau_{2}\right) d \tau_{2} d \tau_{1} \\
& +(d \varepsilon-d) \int_{t-\tau}^{t} \int_{\tau_{1}-\tau}^{\tau_{1}} v_{1}\left(\tau_{2}\right) d \tau_{2} d \tau_{1} \\
& -\varepsilon\left(-\int_{t-\tau}^{t} \int_{\tau_{1}-\tau}^{\tau_{1}} y\left(\tau_{2}\right)^{3} d \tau_{2} d \tau_{1}\right. \\
& \left.+\int_{t-\tau}^{t}\left(y\left(\tau_{1}\right)-y\left(\tau_{1}-\tau\right)\right) d \tau_{1}\right) \\
& +(-a \varepsilon+d) \int_{t-\tau}^{t} \int_{\tau_{1}-\tau}^{\tau_{1}} y\left(\tau_{2}\right)^{2} d \tau_{2} d \tau_{1} \\
& -a \int_{t-\tau}^{t}\left(y\left(\tau_{1}\right)^{2}-y\left(\tau_{1}-\tau\right)^{2}\right) d \tau_{1} \\
& +b \varepsilon \int_{t-\tau}^{t} \int_{\tau_{1}-\tau}^{\tau_{1}} y\left(\tau_{2}\right) d \tau_{2} d \tau_{1}-\left(I \varepsilon+b c_{y} \varepsilon\right. \\
& -d+\varepsilon) \tau^{2} .
\end{aligned}
$$

Evaluating this ID-IO polynomial at each $t_{k}, k=1, \ldots, N$ provides a linear system of the form $A \tilde{\Theta}=b$ where $\tilde{\Theta}=\left(-\left(-\varepsilon y_{0}+\varepsilon+y_{0}-1\right),-(d \varepsilon-d), \varepsilon,-(-a \varepsilon+\right.$ $\left.d), a,-b \varepsilon, I \varepsilon+b c_{y} \varepsilon-d+\varepsilon\right)$ and

$$
\begin{aligned}
& b_{k}=z_{k}-2 z_{k-\tau}+z_{k-2 \tau}+I_{1}^{e s t}\left(y(.)^{3}-y(.-\tau)^{3}\right), \\
& A_{k}=\left(I_{2}^{e s t}\left(u_{1}\right), I_{2}^{e s t}\left(v_{1}\right),-I_{2}^{\text {est }}\left(y^{3}\right)\right. \\
& +I_{1}^{\text {est }}(y(.)-y(.-\tau)), I_{2}^{\text {est }}\left(y^{2}\right) \\
& \left.I_{1}^{\text {est }}\left(y(.)^{2}-y(.-\tau)^{2}\right), I_{2}^{\text {est }}(y), \tau^{2}\right)
\end{aligned}
$$

$A_{k}$ and $b_{k}$ represent the $k^{t h}$ line of $A$ and $b$ respectively ( $k$ from 1 to 7 ). System $A \tilde{\Theta}=b$ is solved using the $\mathrm{QR}$ factorization that does not necessitate an initial guess of the unknown parameters.

\section{Parameter estimation}

The trapezoidal formulas are used to estimate the previous integrals. Let $\left(t_{k}\right)_{k=1, \ldots, N}$ the discretization points of the time interval $[0, T], h=T /(N-1)$. Then, for a simple integral, one gets:

$$
\int_{0}^{T} f(s) d s=h\left(\frac{1}{2} f\left(t_{1}\right)+\frac{1}{2} f\left(t_{N}\right)+\sum_{i=2}^{N-1} f\left(t_{i}\right)\right)
$$

and for a double integral

$$
\begin{aligned}
& \int_{a}^{b} \int_{\phi_{1}(t)}^{\phi_{2}(t)} f(s) d s d t=h\left(\frac{1}{2} \int_{\phi_{1}(a)}^{\phi_{2}(a)} f(s) d s\right. \\
& \left.+\frac{1}{2} \int_{\phi_{1}(b)}^{\phi_{2}(b)} f(s) d s+\sum_{i=2}^{N-1} \int_{\phi_{1}\left(t_{i}\right)}^{\phi_{2}\left(t_{i}\right)} f(s) d s\right)
\end{aligned}
$$

For the simulations, the following values are taken: $a=3, b=4, d=5, I=3.25 \mathrm{~A}, \varepsilon=0.12$, $\left(x_{1}(0, \theta), x_{2}(0, \theta), x_{3}(0, \theta)\right)=(0.2 ; 0.7 ; 4)$. The time interval is $[0,100]$ with a step size $h=0.01$ s. The integrals are evaluated in using 29 points. This number gave the best results after several numerical tests. The outputs are perturbed by an additive white centered Gaussian noise with a standard deviation $\sigma$ given by the three successive values: $0.0001,0.0005,0.001$. For each of this value, the estimate of $\hat{a}, \hat{b}, \hat{d}, \hat{\varepsilon}$ are given in Table 1 . Table 2 provides first, the relative errors between the exact and the estimated parameters, that is between $\theta=(\varepsilon, a, b, c)$ and $\hat{\theta}=(\hat{\varepsilon}, \hat{a}, \hat{b}, \hat{c})$ then, the relative errors between the measured output and the simulated output obtained with $\hat{\theta}$.

\begin{tabular}{|c|c|c|c|c|}
\hline$\sigma$ & $\hat{\varepsilon}$ & $\hat{a}$ & $\hat{b}$ & $\hat{d}$ \\
\hline 0.0001 & 0.1204 & 3.0013 & 3.9712 & 4.9757 \\
\hline 0.0005 & 0.1390 & 2.9371 & 3.9790 & 5.0537 \\
\hline 0.001 & 0.15437 & 2.8076 & 4.4480 & 5.1488 \\
\hline
\end{tabular}

Table 1: Parameter values obtained with the method presented at Section II.

\begin{tabular}{|c|c|c|}
\hline$\sigma$ & $\begin{array}{c}\text { Relative errors } \\
\text { on the parameters }\end{array}$ & $\begin{array}{c}\text { Relative errors } \\
\text { on the outputs }\end{array}$ \\
\hline 0.0001 & 0.005 & 0.0161 \\
\hline 0.0005 & 0.012 & 0.1949 \\
\hline 0.001 & 0.072 & 0.4923 \\
\hline
\end{tabular}

Table 2: Relative errors between $\theta$ and $\hat{\theta}$ (that is

$\|\hat{\theta}-\theta\|_{2} /\|\theta\|_{2}$ ) obtained with the method presented at Section II.

The standard deviation of the Gaussian noise is given by a factor of the model output. Model outputs obtained with the different estimated parameter values given in Table 1 are represented at Figure 5.

For the three deviations, the relative error on the estimated parameters is good contrary to the relative error on the outputs. For the two standard deviations 0.0005 and 0.001 , the behavior of the system with the approximated value $\hat{\varepsilon}$ does not correspond to the one with the initial value $\varepsilon$ (for the first one, a longer time interval is required). Indeed, while initial system presents a periodic behavior, the simulated outputs obtained with $\hat{\varepsilon}$ tend to a stable equilibrium point.

Remark 2: The use of the local Levenberg-Marquard algorithm implemented in scilab did not permit to obtain a value of $\varepsilon$ in the accepted behavioral interval in the case of $\sigma=0.001$. Indeed, we obtain the approximated value $\check{\varepsilon}=0.128$ and $\check{\varepsilon}>\varepsilon^{c}>\varepsilon$.

In order to calculate the probability that the approximated value gives the correct behavior of the system, the algorithm given at Section II-C is applied. The results are gathered in Figure 6. For example, for $\varepsilon=0.10,100 \%$ of estimates are in the accepted behavioral interval when $\sigma \leq 0.0002$. If 


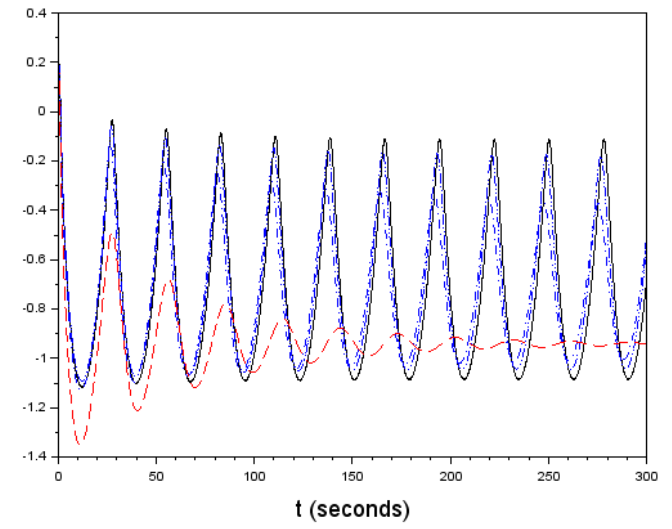

Fig. 5. Outputs of the model obtained with the parameter values given at Table 1. The black solid line represents the measured output. The light blue dash line, dark blue line and red dash line represent respectively the outputs obtained with $\sigma=0.0001, \sigma=0.005$ and $\sigma=0.001$.

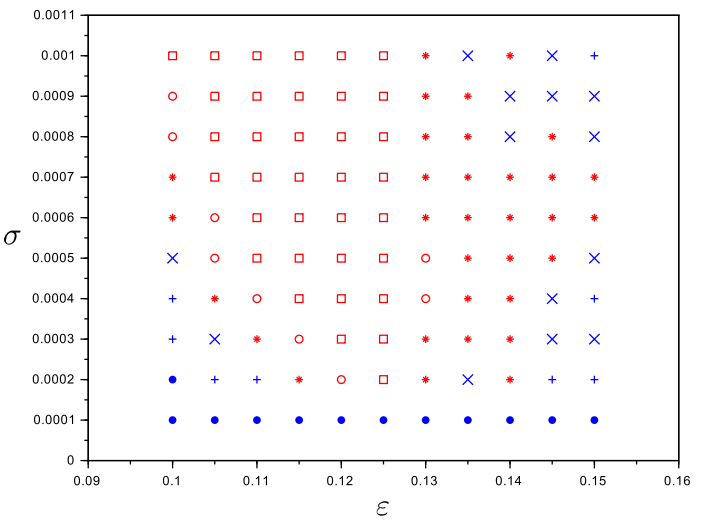

Fig. 6. Percentage of estimated values $\varepsilon$ out of the accepted behavioral interval. In blue points, $0 \%$ of estimated values are out of the accepted behavioral interval. In blue plus, between 0 (excluded) and 5 percents of estimated values are not in the accepted behavioral interval. In blue cross, between 5 and 10 percents of estimated values are not in the accepted behavioral interval. In red asterisk, between 10 and 30 percents of estimated values are not in the accepted behavioral interval. In red circle, between 30 and 50 percents of estimated values are not in the accepted behavioral interval. Finally, in red square, more than $50 \%$ are not in the accepted behavioral interval.

$\sigma \in[0.0003 ; 0.0004[$, less than $5 \%$ of estimated parameters are not accepted behavioral values. Then, the percentage of accepted behavioral values decreases until $\sigma=0.001$ for which more that $50 \%$ of estimated parameters are not accepted behavioral values. For a value $\varepsilon$ equal to 0.12 , as soon as there is some noise, more than $30 \%$ of estimated values are not in the accepted behavioral interval.

\section{CONCLUSION}

The numerical method proposes in this paper permits to obtain a very good first initial guess without any knowledge of the parameter values. However, even if the relative error on the parameters is small, the relative error between the measured outputs and the simulated ones can be important due to a change of the output behavior as seen as for the Hindmarsh Rose model. Indeed, it appears that the effect of the standard deviation influences the expected behavior. More generally, it appears that the consideration of the noise is essential for some particular parametric models since a little error on the parameter values can lead to a completely different behavior of the model. Future works concern the study of i) a similar analysis using other forms of noise, ii) the theoretical evaluations of upper bounds of bias and variance of parameter estimates with respect to intensity of the noise.

\section{REFERENCES}

[1] F. Boulier, A. Korporal, F. Lemaire, W. Perruquetti, A. Poteaux, and R. Ushirobira. An algorithm for converting nonlinear differential equations to integral equations with an application to parameter estimation from noisy data. In Proceedings of Computer Algebra in Scientific Computing 2014, Warsaw, Poland, LNCS, 8660, pages 28-43, 2014.

[2] F. Boulier, D. Lazard, F. Ollivier, and M. Petitot. Computing representation for radicals of finitely generated differential ideals. Technical report, IT-306, Université Lille I, LIFL, 59655, Villeneuve d'Ascq, 1997.

[3] N. Corson, V. Lanza, and N. Verdière. Hopf bifurcations in a chain of coupled hindmarsh-rose system. Acta Biotheoretica, 65(4), 2016.

[4] L. Denis-Vidal, G. Joly-Blanchard, and C. Noiret. Some effective approaches to check identifiability of uncontrolled nonlinear systems. Mathematics and Computers in Simulation, 57:35-44, 2001.

[5] L. Denis-Vidal, G. Joly-Blanchard, C. Noiret, and M. Petitot. An algorithm to test identifiability of non-linear systems. In Proceedings of 5th IFAC NOLCOS, volume 7, pages 174-178, St Petersburg, Russia, 2001.

[6] M Fliess, M Mboup, H Mounier, and H Sira-Ramirez. Questioning some paradigms of signal processing via concret examples. In Proceedings of Summer School: Fast Estimation Method in Automatic Control and Signal Processing, Paris, 2005.

[7] J.L. Hindmarsh and R.M. Rose. A model of the nerve impulse using two first-order differential equations. Nature, 296(5853):162-164, 1982.

[8] J.L. Hindmarsh and R.M. Rose. A model of neuronal bursting using three coupled first order differential equations. Proceedings of the Royal Society of London B: Biological Sciences, 221(1222):87-102, 1984.

[9] A. L. Hodgkin and A. F. Huxley. A quantitative description of membrane current and its application to conduction and excitation in nerve. The Journal of physiology, 117(4):500-544, 1952.

[10] E. M. Izhikevich. Dynamical systems in neuroscience. MIT press, 2007.

[11] S. Kiranyaz, T. Ince, and M. Gabbouj. Optimization Techniques: An Overview. Springer Berlin Heidelberg, 2014.

[12] J.M. Loeb and G.M. Cahen. More about process identification. Automatica, pages 359-447, 1965.

[13] J. Schumann-Bishoff and U. Parlitz. State and parameter estimation using unconstrained optimization. Phys. Re. E, 84, 2011.

[14] H. Sira-Ramirez, C. Garcia Rodriguez, J. Cortes Romero, and A. Luviano Juárez. Algebraic Identification and Estimation Methods. Feedback Control Systems, Wiley, 2014.

[15] E. Steur. Parameter estimation in Hindmarsh-Rose neurons. Technische Universiteit Eindhoren, PhD Thesis, 2006.

[16] I. Tokuda, U. Parlitz, L. Illing, M. Kennel, and Henry Abarbanel. Parameter estimation for neuron models. In AIP Conference Proceedings, volume 676, page 251, 2003.

[17] N. Verdière, S. Zhu, and L. Denis-Vidal. A distribution input-output polynomial approach for estimating parameters in nonlinear models, application to a chikungunya model. Journal of Computational and Applied Mathematics, 331:104-118, 2018.

[18] E Walter and L Pronzato. Identification of parametric models from experimental data. Springer-Verlag, Berlin, 1997. 